\title{
Ungleichheit in Zeiten von Corona oder - Lateinamerikas Wege in die Zukunft
}

\author{
Hans-Jürgen Burchardt
}

Corona hat die menschliche Zivilisation vor enorme Herausforderungen gestellt. Es ist jedoch nicht nur das Virus, das tötet. Covid-19 frisst sich vielerorts in gesellschaftliche Strukturen ein, die bereits durch starke soziale Zerklüftungen gekennzeichnet sind, in denen oft große Teile der Bevölkerung verarmt und soziale Dienste stark fragmentiert sind oder gar nicht existieren. Corona ist nicht nur ein Virus. Es ist auch ein sozialer Marker, der Elend und soziale Ungleichheiten ausleuchtet. Dies trifft insbesondere auf Lateinamerika zu, die Region mit den größten sozialen Ungleichheiten weltweit.

Die Pandemie stellt die lateinamerikanischen Gesellschaften seit ihrem Ausbruch in der Region im Februar 2020 vor extreme Herausforderungen. Es geht nicht nur um eine weitere Krise, es geht um grundsätzliche Fragen: nach dem Entwicklungsmodell, der zukünftigen Rolle des Staates, der Demokratie sowie der Bedeutung der öffentlichen Dienstleistungen und Güter. Die Corona-Pandemie macht deutlich, was systemrelevant ist: ein funktionierendes Gesundheitssystem, eine breite, für allen zugängliche Daseinsvorsorge. Diese Erkenntnis sollte zur Richtschnur für die weitere Entwicklung der lateinamerikanischen Gesellschaften werden (vgl. Burchardt 2021).

Der folgende Beitrag beschreibt mehrere lateinamerikanische Facetten der Pandemie unter der Perspektive sozialer Ungleichheiten. Zuerst wird das soziale Panorama in der Region beschrieben. Auf dieser Basis folgen Empfehlungen, wie die Corona-Pandemie in eine konkrete Politik münden könnte, um die Krise zu überwinden und die Region für die nächste Katastrophe wie z.B. den Klimawandel zu wappnen.

\section{Covid-19 in Lateinamerika: Das soziale Panorama}

Schon 2020 hatte sich Lateinamerika zu einem Pandemie-Hotspot entwickelt. Brasilien und Peru wurden zu komplexen Epizentren. Im weiteren Verlauf kam es zu einer deutlichen Zunahme des Infektionsgeschehens 
auch in so genannten middle income Ländern wie Argentinien, Chile, Mexiko und Kolumbien. Mitte 2021 zählt Lateinamerika offiziell fast 35 Millionen Infizierte und mehr als 1,2 Millionen Tote, mehr als die Hälfte davon alleine in Brasilien. Die Region repräsentiert zwar nur 8,4 Prozent der Weltbevölkerung, stellt aber nach Angaben internationaler Organisationen gut ein Viertel der globalen Todesopfer. Allerdings ist bis heute unklar, wie aufschlussreich die veröffentlichten Zahlen über Infektionen und Todesfälle in der Region sind. Es kann davon ausgegangen werden, dass die offiziellen Zahlen nur ein sehr unvollständiges Bild des Infektionsgeschehen wiedergeben. Ein Beispiel: In Mexiko hat sich nach Studien der Regierung bis Mitte 2021 rund ein Viertel der Bevölkerung mit dem Coronavirus infiziert; das wären mehr als 30 Millionen Mexikaner und Mexikanerinnen. Die Zahl der bestätigten Infektionen beläuft sich aber nur auf rund 2,5 Millionen Menschen. Die offizielle Zahl der Todesopfer liegt bei knapp 230.000. Hier dürfte die tatsächliche Zahl nach Schätzungen mindestens 60 Prozent höher sein (Cham et al. 2021).

Ein Grund für diese hohen Schwankungen sind die begrenzten Testkapazitäten auf dem Land sowie in den armen Stadtvierteln. Ländliche Gebiete sind in Lateinamerika traditionell von extremer Armut und fehlender Gesundheitsversorgung gekennzeichnet. In großen Städten hingegen existiert das Phänomen einer Hyper-Urbanisierung mit hoher Bevölkerungsdichte und geringen hygienischen Standards; dafür gibt es häufig Gesundheitsbelastungen durch starke Schadstoffkonzentrationen. Nach verschiedenen Studien lebt ein Fünftel der lateinamerikanischen Bevölkerung in informellen oder prekären urbanen Siedlungen, in denen es oft an allem fehlt. Häufig gibt es zu wenig sanitäre Versorgung sowie unzureichende Wasser-, Energie- und Stromversorgung. Viele Wohnungen sind unangemessen ausgestattet und mit mehr als drei Personen pro Schlafraum belegt. Der öffentliche Nahverkehr ist prekär und beengt. Ideale Voraussetzungen für die Verbreitung des Virus. All dies erschwert in vielen Ländern sowohl die Erfassung und Nachverfolgung von Infektionen wie auch die Seuchenbekämpfung. Dazu kommen in manchen Ländern politische Befindlichkeiten wie die Verharmlosung des Virus in Brasilien oder das Leugnen von Infektionsdynamiken wie in Nicaragua (s. Vestena und Krawinkel in diesem Band).

Weiß man in Lateinamerika zu wenig über das reale Infektionsgeschehen, sind die Gründe für die Verbreitung des Virus seit langem wohlbekannt: dazu zählen als erstes die mit Personal und Ressourcen zu schwach ausgestatteten, oft teilprivatisierten und darum signifikant fragmentierten Gesundheitssysteme der Region. Allein die Schätzungen der panamerikanischen Gesundheitsorganisation Pan Amercian Health Organization (PA- 
$\mathrm{HO})$ verdeutlichen dies eindrücklich: Die PAHO hält eine Bereitstellung von 6 Prozent des regionalen Bruttoinlandsprodukts (BIP) für erforderlich, um in Lateinamerika eine universelle solide Gesundheitsversorgung zu gewährleisten. Die meisten Länder investieren aber durchschnittlich nur 2,4 Prozent des BIP in die Gesundheit; die einzigen Ausnahmen sind Kuba, Argentinien und Costa Rica (CEPAL 2019a: 139-140).

Zusätzlich leidet die Bevölkerungsmehrheit Lateinamerikas unter prekären Einkommensverhältnissen, die die soziale Krise weiter verschärfen, aber auch die Einhaltung von Pandemievorschriften erschweren: 2019 verfügten 77 Prozent der lateinamerikanischen Bevölkerung (470 Millionen Menschen) über ein niedriges oder mittleres Einkommen. Diese Mehrheit hat kaum Ersparnisse, um die aktuellen Krisenkosten abzufedern (CEPAL 2019b; 2020a). Die Internationale Arbeitsorganisation International Labour Organization (ILO) befürchtet, dass zusätzlich mehr als die Hälfte der formell Beschäftigten der Region von Arbeitsplatzverlusten betroffen sein wird (ILO 2020a: 14). Viele dieser von Arbeitslosigkeit Bedrohten haben kaum oder keinen Anspruch auf Sozialversicherungen oder Renten. Nur elf der 33 Länder in der Region kennen überhaupt eine Arbeitslosenversicherung und ein Drittel der erwerbstätigen Personen, die in Haushalten mit niedrigem und unterem bis mittlerem Einkommen leben, ist rentenversichert (CEPAL 2020b). Noch schlimmer ist Lage für die informell Beschäftigten, also über 50 Prozent aller Erwerbstätigen. Hier ist die Pandemie meist existenzbedrohend: Die regelmäßigen Lockdowns und anderen Auflagen zur Sicherung physischer Distanz führen zu signifikanten Beschäftigungs- sowie massiven Einkommensverlusten. Dies betrifft über 160 Millionen Personen; viele von ihnen sind junge Menschen und Frauen, die auf der Suche nach einem täglichen Broterwerb durch die Straßen ziehen (ILO 2020b; vgl. Weller in diesem Band). Die Mehrheit dieser Schutzbedürftigen können hygienische Mindeststandards nicht einhalten, grundlegende Maßnahmen wie Händewaschen oder Vermeiden von Körperkontakt sind für sie schwierig oder unmöglich. Social distancing ist nicht durchsetzbar und würde den Hungertod bedeuten.

Die Krise trifft die Armen und Vulnerablen also am stärksten. Für sie bedeutet nicht nur das Virus, sondern auch ein Lockdown oft Elend oder im Extremfall sogar Tod. Wie in den meisten Ländern des Globalen Südens ist auch in Lateinamerika das pandemiebedingte "zu Hause bleiben“ ein Luxus, den sich nur eine Minderheit leisten kann.

Die Armutszahlen der Region lassen jetzt schon erahnen, welche schwere soziale und wirtschaftliche Krise die Pandemie provozieren wird: Laut der UN-Wirtschaftskommission für Lateinamerika (Comisión Económica para América Latina y el Caribe, CEPAL) waren vor der Pandemie 2018 
rund ein Drittel der Bevölkerung in der Region arm, fast die Hälfte dieser Armut konzentrierte sich auf die ländlichen Gebiete. Vor allem unter von Arbeitslosigkeit und Nichterwerbstätigkeit betroffenen Menschen ist die Armut hoch. In dieser Gruppe gelten 41,6 bzw. 29,8 Prozent als arm, gefolgt mit 29,4 Prozent Armut bei den sogenannten "Selbstständigen und unbezahlten Familienmitgliedern" (CEPAL 2019c: 19). Seit der Pandemie ist diese Armut sprunghaft angestiegen und wird nach allen Prognosen weiter steigen. Eng verbunden mit der Armut ist das dramatische Ansteigen der sozialen Ungleichheit. Während es hier in den ersten 15 Jahren dieses Jahrhunderts in der Region eine diskrete, aber doch sichtbare Verringerung gab, hat sich diese Dynamik mit der Pandemie vollständig umgekehrt: Ungleichheiten steigen wieder stark an.

Tabelle: Lateinamerika (17 Länder): Prognostizierte Veränderung des GINI-Index im Jahr 2020

\begin{tabular}{cccc}
\hline $1,0 \%$ bis $2,9 \%$ & $3,0 \%$ bis $4,9 \%$ & $5,0 \%$ bis $6,9 \%$ & Mehr als $7,0 \%$ \\
\hline Guatemala & Bolivien (Plurinationaler Staat) & Brasilien & Argentinien \\
\hline Honduras & Costa Rica & Chile & Ecuador \\
\hline Nicaragua & Panama & Kolumbien & Peru \\
\hline Paraguay & Dominikanische Republik & El Salvador & \\
\hline & & Mexiko & \\
\hline & Uruguay \\
\hline
\end{tabular}

Quelle: CEPAL (2021a: 44).

Viele Beobachterinnen und Beobachter gehen davon aus, dass sich die sozialen Zerklüftungen in der Region mit und nach der Pandemie weiter vertiefen und für große Bevölkerungsschichten lang anwachsende soziale und wirtschaftliche Unsicherheiten provozieren. Die CEPAL (2020a) befürchtet, dass Lateinamerika im Feld der sozialen Entwicklung um mehr als ein Jahrzehnt zurückfallen wird. Covid-19 raubt also nicht nur vielen Menschen die Gesundheit oder das Leben. Der Virus hat auch das Potenzial, die Gesellschaften Lateinamerikas über Jahre hinaus zu schwächen oder sogar zu zerreißen. Dies stellt auch für die relativ gefestigten Demokratien der Region eine wachsende Bedrohung dar. Und schlimmer: Armut und Misere sind ein exzellenter Nährboden für das Virus. In lokalen Räumen kann es gut gedeihen, sich weiterentwickeln und sich mit neuen Mutationen in immer neuen Wellen in ganz Lateinamerika und der Welt ausbreiten. 


\section{Von Corona lernen: Wege in die Zukunft}

Wenn die lateinamerikanischen Gesellschaften die aktuelle Covid-19-Krise überwinden und zusätzlich verhindern wollen, dass die nächste Pandemie oder die Klimakrise die Region in gleicher Weise oder sogar noch härter treffen, müssen sie von Corona lernen. Die Lektion ist einfach: Die sozialen Ungleichheiten sind entschlossen zu verringern und zwar nicht nur durch neue Kampagnen zur direkten Bekämpfung von Armut. Armut ist immer eine Frage von Ungleichheit. Das Phänomen der persistenten sozialen Ungleichheit ist in Lateinamerika gut erforscht und ausgeleuchtet. Ursachen und Lösungen zur Überwindung der eklatanten Ungleichheiten sind seit langem bekannt, aber bisher fehlte es am politischen Willen die nötigen Strukturreformen einzuleiten. Dieser Befund trifft auch auf die progressiven Regierungen zu, die ab Ende der 1990er Jahre angetreten waren, um die soziale Frage zu bearbeiten (Burchardt 2020).

Bei einer genaueren Beschäftigung mit sozialer Ungleichheit in der Region wird deutlich, dass sich nicht nur die Oberschicht, sondern auch die Mittelschichten weitgehend mit den existierenden Ungleichheiten gut arrangiert haben und oft von ihnen profitieren. In Lateinamerika erwirtschaften gerade einmal 20 Prozent der Erwerbstätigen rund 40 Prozent des regionalen BIP. Diese Minderheit ist ökonomisch gut abgesichert und verfügt über einen Zugang zu exzellenten sozialen Dienstleistungen. Die mehr als 50 Prozent informellen Arbeitskräfte sind ökonomisch irrelevant, ihr Anteil am regionalen BIP beträgt gerade einmal 10 Prozent (CEPAL 2012). Mit anderen Worten: sie sind für die Entwicklung der Region gesamtwirtschaftlich kaum erforderlich.

Dieses Segment an prekär Arbeitenden und Lebenden hilft den Mittelund Oberschichten hingegen ganz entschieden, ihr Leben noch komfortabler zu gestalten. So ist die wenig regulierte und schlecht bezahlte Hausarbeit seit langem einer der wichtigsten Stützen für das gute Leben der lateinamerikanischen Mittelschichten. Nach Südostasien werden in Lateinamerika weltweit die meisten Hausangestellten beschäftigt: Im Jahr 2016 machten 18 Millionen Pflegekräfte 7 Prozent der regionalen Erwerbstätigen aus, mehr als 90 Prozent davon waren Frauen und fast 80 Prozent waren informell beschäftigt, also ohne Arbeitsvertrag, ohne Sozialversicherung und mit niedrigen Löhnen (ILO 2016). Darüber hinaus sind Indigene und Schwarze in dieser Gruppe stark überrepräsentiert (Lextartza et al. 2016; zu Hausarbeit s. Weinmann 2020).

Mit Blick auf diese Strukturmerkmale der lateinamerikanischen Gesellschaften lassen sich drei Schritte identifizieren, mit denen der Verschär- 
fung der Ungleichheit im Kontext der Corona-Krise in Lateinamerika begegnet werden kann.

\section{Ausweitung der Daseinsvorsorge}

Corona ist die Stunde für soziale Reformen. In Pandemiezeiten ist es am dringlichsten, die geschwächten und dysfunktionalen Gesundheitssysteme zu stabilisieren, um die Kranken adäquat behandeln und versorgen, sowie die Ausbreitung der Infektion eindämmen zu können. Daher sollten in einem ersten Schritt alle notwendigen Ressourcen mobilisiert werden, um die Gesundheitssysteme auf den international empfohlenen Standard zu bringen. Hierzu ist vor allem eine Aufstockung mit Personal erforderlich: Die Weltgesundheitsorganisation World Health Organization (WHO) (2017) schätzt, dass weltweit ein Minimum von 3,0 Personen im Gesundheitspersonal pro 1.000 Einwohner notwendig ist, um das Ziel Drei der nachhaltigen Entwicklungsziele (Sustainable Development Goals, SDGs) der Vereinten Nationen „Gesundheit und Wohlbefinden“ zu erreichen. In Lateinamerika wird diese empfohlene WHO-Personaldichte nur von drei Ländern erreicht: Argentinien $(3,96)$, Kuba $(8,4)$ und Uruguay $(5,05)$.

Doch im Gesundheitswesen geht es nicht nur um Ressourcen. Brasilien und Chile sind gute Beispiele dafür, dass sich die Qualität eines Gesundheitssystems nicht nur über hohe Ausgaben definiert, sondern auch über eine institutionelle Ausrichtung, die ein breite Gesundheitsvorsorge garantiert. In den beiden genannten Ländern sind die Gesundheitssysteme prinzipiell gut ausgestattet und verfügen über hinreichende Ressourcen. Sie zeichnen sich aber gleichzeitig durch eine starke vertikale (hauptsächlich städtische) Struktur aus, bei der es sehr gute, meist private Dienstleistungen für wenige, statt einer universellen Vorsorge und Betreuung für alle gibt. Corona hat dieses System zusammenbrechen lassen, indem es auch die exklusiven privaten Dienstleister überlastete. Die Erfahrungen in Kuba und Uruguay unterstreichen, dass ein Gesundheitswesen mit universeller Abdeckung, robuster Ausstattung und ausreichend Personal in der Lage ist ein Krisenszenario wie die Pandemie besser zu bewältigen. Es geht in Zukunft darum, den Zugang zur Gesundheitsversorgung zu erweitern; ein Schwerpunkt sollte hierbei in den städtischen Armutsvierteln und im ländlichen Raum liegen.

Corona macht als sozialer Marker weiterhin deutlich, dass sich die Pandemiebekämpfung nicht auf eine Verbesserung der Gesundheitsversorgung oder auf einen Impfstoff beschränken darf, sondern generell die miserablen Lebens- und Arbeitsbedingungen reduzieren muss. In den 
Ländern mit schwachen Gesundheitssystemen, großer Armut und einem hohen Anteil informeller Wirtschaft und Arbeit bleibt als erste Notmaßnahme sicherlich nur die Option auf Sofort- und Direkthilfen für die Grundsicherung der gefährdeten Bevölkerung. Die Erfahrungen der letzten zwei Jahrzehnte mit der Umsetzung von Bargeldtransfers, den so genannten cash transfers wie in Brasilien, haben gezeigt, dass solche Maßnahmen ebenso billig wie effektiv sind: Sie erreichen direkt gefährdete soziale Gruppen, fördern deren materielle Existenzsicherung und haben geringe Kosten (im Durchschnitt weniger als 1 Prozent des nationalen BIP bzw. 0,25 Prozent des regionalen BIP) (Blofield/Filgueira 2020; CEPAL 2016, 2020b). Zur Stabilisierung der Region darf man aber bei solchen assistentialistischen Maßnahmen nicht stehen bleiben. Sie müssen in einen zügigen Ausbau einer breiten Daseinsvorsorge münden. Die Rede ist von einer flächendeckenden Versorgung mit sozialen Dienstleistungen für alle, die gleichzeitig als soziale Rechte abgesichert sind. Das Ziel muss es also sein für alle systemrelevanten Bereiche eine möglichst universelle öffentliche Infrastruktur aufzubauen.

Die CEPAL (2020a: 19) bringt hierbei als eine der wichtigsten Maßnahmen eine Einkommensgarantie über ein universelles Grundeinkommen in Spiel:

„Die zentralen Aktionen sind die Einrichtung einer universellen Einkommensgarantie, insbesondere für informelle und prekäre Arbeiter sowie der universelle Zugang zu Tests und medizinischer Versorgung für alle, die sie benötigen. Dazu gehören grundlegende Dienstleistungen, Wohnraum, angemessene Ernährung und Bildung für Kinder, Jugendliche und junge Erwachsene. Mittel- und langfristig sollte die soziale Absicherung zu einer zentralen Politik für den Abbau von Ungleichheiten, für die Förderung von sozialer Inklusion und integrativen Wachstums und damit für den sozialen Zusammenhalt werden. Dafür ist es entscheidend, universelle soziale Schutzsysteme zu konsolidieren, einschließlich universeller Gesundheitssysteme, die auf einem Rechtsanspruch basieren und für Unterschiede sensibel sind. Zusätzlich sollten in der Erholungsphase Strategien für die Integration der Arbeitskräfte entwickelt werden“ (Übersetzung des Autors).

Insofern sind massive Investitionen in die Gesundheitsinfrastruktur, die Pflege, die Bildung, die Grundversorgung, die zivile und öffentliche Sicherheit, den städtischen öffentlichen Nahverkehr, die Förderung der ländlichen Gebiete und der lokalen Wirtschaft erforderlich. Innerhalb dieser Maßnahmen müssen die reproduktiven Tätigkeiten der Pflege und Betreuung strukturell, aber auch kulturell besonders aufgewertet werden. Die 
Pandemie hat weltweit deutlich gemacht, dass Sorgearbeit systemrelevant ist. Hierbei wurden auch die geschlechtsspezifischen Schieflagen sichtbar: Fast drei Viertel aller Beschäftigten im Gesundheits- und Pflegesektor (formell und informell) in Lateinamerika sind Frauen. Sie verdienen im Durchschnitt 25 Prozent weniger als Männer und sind gleichzeitig aufgrund der geschlechtsspezifischen Arbeitsteilung einem deutlich höheren Infektionsrisiko ausgesetzt (Kessler/ Benza 2020).

Maßnahmen zum Ausbau der öffentlichen Daseinsvorsorge würden sich schnell selbst tragen und legitimieren: Wenn Ausgaben bei Pflegediensten oder Bildung nicht mehr in der Verantwortung des Einzelnen liegen, sondern mit breitem Zugang und in hoher Qualität vom Staat gewährleistet werden, entscheiden sich viele Familien sicherlich nicht mehr für private Krankenversicherungen, versuchen Steuern zu vermeiden oder beschäftigen Hausangestellte informell und zu unwürdigen Bedingungen. Schließlich liegt es vor allem am hohen Anteil billiger Hausarbeit, dass es selbst bei ausreichend vorhandenen gesellschaftlichen Ressourcen in der Region bisher nicht erforderlich - oder durchsetzbar - war ein System hochwertiger öffentlicher Dienstleistungen zu schaffen, welches für die Mehrheit zugänglich ist. Eine Formalisierung - und somit Verteuerung von Hausarbeit gekoppelt mit einer Neubewertung und staatlicher Förderung systemrelevanter Tätigkeiten in Pflege, Betreuung und Bildung wird die Entstehung eines öffentlichen Gemeinwohls signifikant unterstützen (dies trifft genauso auf Deutschland zu: Burchardt 2021).

\section{Reduzierung der informellen Arbeit}

Bei einer genaueren Analyse des lateinamerikanischen Infektionsgeschehens wird deutlich, wie stark die Existenz der informellen Ökonomie die Pandemiebekämpfung ausbremst oder einzelne Maßnahmen sogar ganz verhindert (ILO 2020a). Für viele prekär Erwerbstätige würde die Umsetzung aller pandemiebedingten Schutzmaßnahmen Hunger, Elend oder gar Tod bedeuten. Die informelle Arbeit wurde in Lateinamerika - sowie in großen Teilen des Globalen Südens - zum Brandbeschleuniger der Pandemie. Corona lehrt uns also, dass die Stärkung der gesellschaftlichen Krisenresilienz eine signifikante Reduzierung von informeller Arbeit erfordert:

„Die COVID-19-Krise hat einmal mehr die Verwundbarkeit der Millionen von Menschen offengelegt, die ihren Lebensunterhalt in der informellen Wirtschaft verdienen und erinnert daran wie wichtig es ist den Übergang von der informellen zur formellen Wirtschaft zu einem 
Schwerpunkt der nationalen Politik zu machen. Ein wirtschaftlicher Aufschwung ist zwar notwendig, wird aber allein die Informalität nicht verringern; eine adäquate öffentliche Politik ist ebenfalls unerlässlich“ (ILO 2020a: 8, Übersetzung des Autors).

Doch die Existenz eines breiten Sockels informeller Arbeit erschwert nicht nur die Seuchenbekämpfung. Sie hat weitere beachtliche Auswirkungen auf die regionalen Volkswirtschaften, auf die sozialen und politischen Konstellationen sowie auf die Notwendigkeit der anstehenden sozial-ökologischen Transformation zur Eindämmung des Klimawandels und massiven Verlusts biologischer Vielfalt in der Region. Die Mehrheit der gering qualifizierten Arbeitsplätze steht nicht nur im Gegensatz zum Aufbau einer breit angelegten Sozialpolitik. Sie ist gleichzeitig das Haupthindernis für die Steigerung der Arbeitsproduktivität und blockiert somit die für Lateinamerika strategisch bedeutsame Option, über eine erhöhte Wertschöpfung durch Arbeit und Wissen die umweltschädliche Ausbeutung und den Export von Rohstoffen (wie Öl, Gas, Bergbau, aber auch Agrarprodukte) zu verringern.

So war selbst während des enormen Wirtschaftsbooms der letzten zwei Jahrzehnte in der Region kein Anstieg der Arbeitsproduktivität oder des Anteils der Lohnsumme am BIP (der den Anteil der nationalen Wertschöpfung durch Arbeit misst) zu verzeichnen (Burchardt et al. 2016). Nach Angaben der ILO (2013) ist die Arbeitsproduktivität in Lateinamerika nahezu unverändert halb so hoch wie der globale Durchschnittswert. Der Anteil der Lohnsumme am regionalen BIP ist sogar gesunken:

„Die Arbeitsproduktivität, gemessen als produziertes BIP pro Arbeitsstunde, ist in den letzten zehn Jahren in Lateinamerika im Vergleich zu anderen, weiterentwickelten Ökonomien zurückgegangen. Im Jahr 2016 erreichte die Arbeitsproduktivität in Lateinamerika ein Drittel der USA-Durchschnitts, ein geringerer Anteil als noch vor 60 Jahren. Dies steht in krassem Gegensatz zur Entwicklung der wachstumsstarken asiatischen Länder wie Korea und in jüngerer Zeit China oder auch der Rohstoffexporteuren wie Australien, deren relative Produktivität stabil blieb“ (OECD et al. 2016: 65, Übersetzung des Autors).

Eine postpandemische Politik in der Region muss sich darum besonders auf eine Verringerung der informellen Ökonomie konzentrieren. Die dafür zu ergreifenden politischen Maßnahmen sind erforscht und bekannt (Burchardt et al. 2013): Erstens muss die traditionelle Politik, sich bei Sozialreformen auf eine vertikale Verbesserung der sozialen Dienstleistungen zu fokussieren, in eine Horizontalisierung, also eine Universalisierung der 
Sozialleistungen münden. Konkreter: Heute ist die soziale Absicherung in Lateinamerika überwiegend den Angestellten wichtiger strategischer, meist formeller Sektoren vorbehalten, während die ärmsten 20 Prozent der Region nur 10 Prozent aller Sozialtransfers erhalten. Um diese Konstellation zu ändern, ist eine horizontale Ausweitung des Abdeckungsgrades der sozialen Dienste erforderlich, der gefährdete und/oder ausgegrenzte Bevölkerungsgruppen stärker einbezieht, insbesondere im Bereich der informellen, weiblichen und ländlichen Arbeit. Zweitens zeigen die Beispiele Brasiliens und anderer Länder im letzten Jahrzehnt, dass die alleinige Formalisierung der Beschäftigung durch Verträge nicht bessere Arbeitsbedingungen garantiert (Hecker 2020). Die Arbeitsmarktpolitik muss gleichzeitig die Qualität der Arbeitsbeziehungen verbessern, also Maßnahmen verfolgen, die sich auf Arbeitszeiten, Lohnstruktur, Arbeitsschutz usw. konzentrieren. Drittens arbeiten die durch die Pandemie am meisten gefährdeten Menschen in der informellen Wirtschaft. Die Rede ist von Frauen, Jugendlichen, Landarbeiterinnen und -arbeitern sowie ethnischen Gruppen, deren Arbeit nicht nur systemrelevant, sondern oft auch unsichtbar ist, da sie weder in Statistiken noch in der Politik auftauchen.

Zusätzlich kann die Politik diese Maßnahmen für ein Post-Corona-Szenario nutzen, welches die nachhaltige Steigerung der regionalen Arbeitsproduktivität und somit eine ebenso effiziente wie ökologisch verträgliche Wirtschaft zum Ziel hat. Auch in Lateinamerika sollte in Zukunft die Schaffung von Wohlstand nicht mehr primär von der Ausbeutung der Natur abhängen, sondern von Arbeit und Wissen. Menschenwürdige Arbeitsbedingungen durch sozialen Dialog, sozialen Schutz, Mitbestimmung sowie Rechtssicherheit und eine Ökologisierung der Wirtschaft sind hierfür erforderlich. Eine solche arbeits- und wirtschaftspolitische Ausrichtung würde nicht nur den Anforderungen des Klimawandels und nachhaltigerer Entwicklungsmodelle gerecht werden, sondern auch die verschiedenen sozial benachteiligten Gruppen in die Gesellschaft integrieren. Dieser Schritt ist unumgänglich, wenn nach der Pandemie die viel größere Herausforderung des Klimawandels in Lateinamerika bewältigt werden soll.

\section{Faire Lastenverteilung - gerechte Gesellschaft}

Die übliche Antwort auf die hier genannten Vorschläge - die zu Pandemiezeiten eigentlich Verpflichtungen sind - kennen alle: Wer soll das zahlen? Staat und Unternehmen haben doch nur begrenzte Mittel, um die millionenschweren Investitionen, die eine universelle Daseinsvorsor- 
ge oder die Verbesserung der Arbeitsbedingungen bedeuten würden, zu schultern? Mitnichten!

Corona zeigt uns, dass fehlende Entwicklung oder Armut keine Frage von Ressourcenknappheit ist, sondern vor allem in einer ungleichen Verteilung begründet liegt. Nicht nur die Armut ist seit Corona massiv angestiegen, auch der Reichtum: Mit Pandemiebeginn gab es allein 2020 in der Region alle zwei Wochen einen weiteren Milliardär, während schätzungsweise bis zu 52 Millionen Menschen im gleichen Jahr arm wurden und 40 Millionen ihre Arbeit verloren. Das Vermögen der Supermillionäre ist allein bis März 2020 um 17 Prozent gewachsen: 48,2 Mrd. US-Dollar, das entspricht 38 Prozent aller Konjunkturpakete, die die Regierungen Lateinamerikas im gleichen Zeitraum aktiviert haben, und ist neunmal so hoch die Notkredite des Internationalen Währungsfonds IWF für die Region (Ruiz 2020: 3).

Wenn man anerkennt, dass die Corona-Pandemie nur durch gemeinsame Anstrengungen überwunden werden kann, also durch Gemeinsinn und kollektive Verantwortung, wird klar: Sie ist nur durch eine faire Umverteilung zu beenden. Das Gebot der Stunde sind darum nicht nur Sozialund Arbeitsreformen, sondern zusätzlich eine regionale Steuerreform, die die Krisenlasten auf alle Schultern verteilt. Hier ist einiges zu tun. Bis heute ist Lateinamerika eine der Regionen mit den niedrigsten Steuersätzen der Welt und somit eine Oase für Reiche: In einigen Ländern beträgt die Vermögenskonzentration 60 Prozent des nationalen BIP; auf diese gigantischen Vermögen werden kaum Steuern gezahlt (Alstadsæter 2018; Martorano 2018). In der Region entsprechen die staatlichen Einnahmen aus der Vermögenssteuer 1,8 Prozent des BIP; im Vergleich dazu liegen sie in der OECD bei 8,3 Prozent (ECLAC 2018: 8). Auch die progressiven Regierungen der letzten zwei Jahrzehnte haben es in keinem einzigen Land der Region geschafft, ein faires Steuersystem zu etablieren, welches die hohen Einkommen und großen Vermögen in die gesellschaftliche Pflicht nimmt (OECD 2019). 
Abbildung: Steuereinnabmen in Prozent des BIP, Lateinamerika und Karibik und OECD im Vergleich 1990-2019

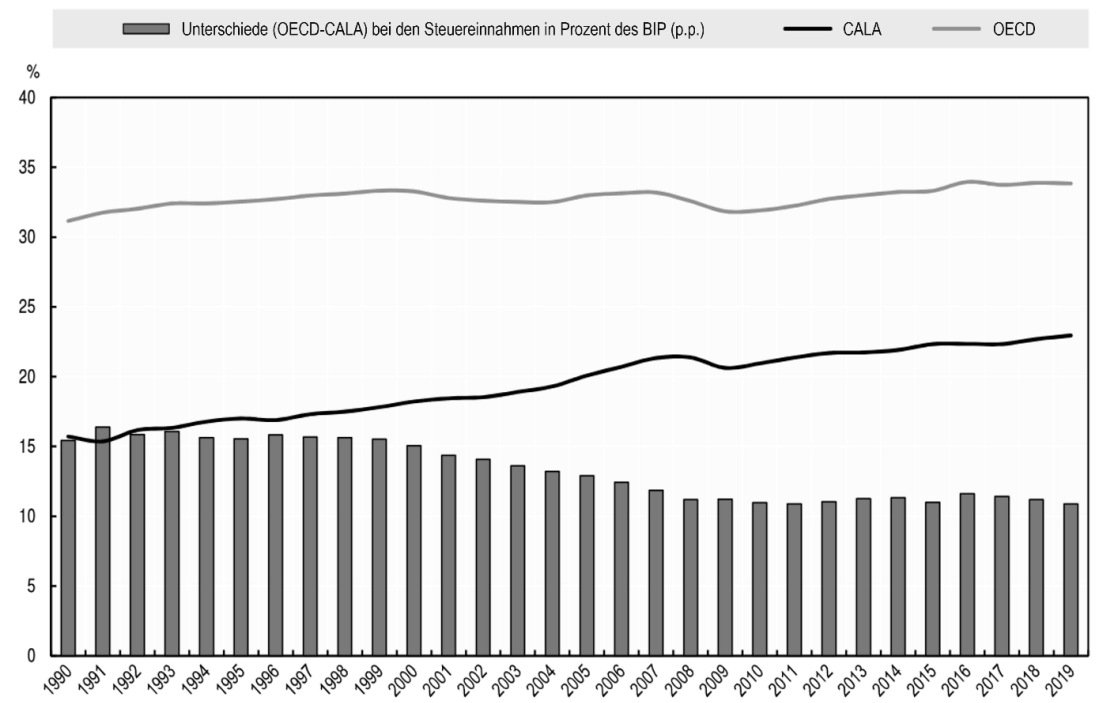

Quelle: OECD (2021: 71).

Eine Steuerreform, die effektiv höhere Einkommen und Vermögen besteuert, ist nicht nur eine Frage technischer Umsetzung, wie es in vielen nationalen Institutionen und internationalen Organisationen angenommen wird. Es geht vielmehr darum, Steuerlegitimität zu schaffen, d.h. die Ablehnung gegenüber Steuern bei wichtigen Teilen der Gesellschaft zu verringern. Dies lässt sich nicht nur mit Gesetzen oder effizienten Verwaltungen erreichen, sondern mit einem Staat, der hochwertige öffentliche Güter anbietet, die breit zugänglich sind. Eine Daseinsvorsorge, die nicht nur hilft die Pandemie kurzfristig zu überwinden, sondern auch garantiert, dass sich langfristig die Lebensqualität der Steuerzahler erhöht.

Corona bietet die Gelegenheit diese Legitimität zu erzeugen und dabei auch die reichsten 10 Prozent der Region zu einem Steuerbeitrag zu verpflichten. Denn viele von ihnen mussten lernen, dass man dem Virus nicht mit privaten Krankenversicherungen, durch die hohen Mauern von Gated Communities oder mit einer Flucht in den USA entkommen konnte.

Was Lateinamerika jetzt braucht ist eine gute, über Steuern finanzierte soziale Versorgung und ein Gemeinwohl für alle und mit allen.

Die regionalen Steuerreformen der letzten Jahrzehnte waren sowohl in Bezug auf die Gesamtsteuerlast als auch auf die Steuerkapazität wenig 
zufriedenstellend: Mit einem durchschnittlichen Steueraufkommen von 23 Prozent des BIP im Jahr 2018 ist die Region weit von dem Durchschnitt der OECD-Länder mit 34,3 Prozent entfernt. Insgesamt lässt sich sagen, dass die Steuersysteme in Lateinamerika stark regressiv ausgerichtet sind und/oder nicht genügend Ressourcen mobilisieren, um die aktuelle Gesundheits- und Wirtschaftskrise zu überwinden und eine inklusive und nachhaltige Entwicklung zu finanzieren.

Ein ausgewogenes Steuersystem kann politisch durch folgende Maßnahmen wirksam umgesetzt werden: Die Abschaffung pauschaler Steuerbefreiungen für hohe Einkommen und Vermögen könnte je nach Land zusätzliche Einnahmen zwischen 2 und 6 Prozent des BIP generieren (CEPAL/Oxfam 2019: 24). Bei der Einkommenssteuer gibt es viel Spielraum: Die Spitzensteuerbelastung liegt bei 25-40 Prozent, bei Finanzund Kapitaleinkommen sogar nur bei 5-15 Prozent; eine Vermögenssteuer haben derzeit nur Argentinien, Uruguay und Kolumbien (CIAT 2018). Insgesamt zahlen die oberen 10 Prozent der Einkommen in Lateinamerika einen durchschnittlichen effektiven Steuersatz von nur 5,6 Prozent auf ihr Einkommen, in einigen Fällen sogar nur 1-3 Prozent (Amarante/Jimenez 2016: 62). Mit dem öffentlichkeitswirksamen Einsatz von Steuergeldern, zum Beispiel für den Bau von Krankenhäusern, kann die Steuerlegitimität auch bei den wirtschaftlichen Eliten Lateinamerikas erhöht werden (für weitere Vorschläge für neue Steuerreformen siehe auch: Ruiz 2020). Insofern stimmt es auch für Lateinamerika: Genug Ressourcen sind da. Es geht nur um ihre Mobilisierung und kluge Verteilung. Corona ist die Stunde der Politik.

\section{Schluss: Die Zeit von Corona ist die Zeit der Veränderung!}

Nach der Krise ist vor der Krise! Die Schockwellen, mit denen das Coronavirus das zersplitterte Lateinamerika zum Knirschen bringt, garantieren keine Veränderung. Krisen sind Prozesse, in denen soziale, ökonomische, kulturelle und politische Konstellationen erschüttert werden oder zerbrechen. Sie bieten die Chance auf neue Konstellationen; aber ebenso können die bestehenden Verhältnisse gestärkt werden. Wenn Lateinamerika nach der Krise in die alten Muster zurückfällt, werden bald die Banken und die großen Unternehmen wieder bestimmen. Dann werden eine neue Austeritätspolitik und Spardiktate die Oberhand gewinnen, die mehr Menschen als Corona das Leben nehmen. Die sozialen Dienste werden weiter abgebaut und bieten immer weniger Schutz. Wenn die nächste Pandemie - oder die Klimakrise, die ebenfalls keine Grenzen kennt - diese letzten 
Reste der Menschheit treffen, ist es unwahrscheinlich, dass sich erneut Chancen für Veränderungen bieten.

\section{Literatur}

Alstadsæeter, Annette; Johannesen, Nils; Zucman, Gabriel (2018): Who owns the wealth in tax havens? Macro evidence and implications for global inequality, in: Journal of Public Economics, 162, 89-100.

Amarante, Veronica; Jiménez, Juan Pablo (2016): Distribución del ingreso e imposición a las altas rentas en América Latina, in: Cuadernos de Economía, 67, 39-73.

Blofield, Merike; Filgueira, Fernando (2020): COVID-19 and Latin America: Social Impact, Policies and a Fiscal Case for an Emergency Social Protection Floor, Buenos Aires: CIPPEC.

Burchardt, Hans-Jürgen; Peters, Stefan; Weinmann, Nico (2013): Arbeit in globaler Perspektive: Facetten informeller Beschäftigung, Frankfurt: Campus-Verlag.

Burchardt, Hans-Jürgen; Domínguez, Rafael; Larrea, Carlos; Peters, Stefan (2016): Nada dura para siempre: Neo-extractivismo tras el boom de las materias primas, Quito: UASB-ICDD.

Burchardt, Hans-Jürgen (2020): Lateinamerikas Rechtsruck: Zur Verantwortung der progressiven Regierungen, in: Eser, Patrick; Witthaus, Jan-Henik (Hg.): Rechtswende in Lateinamerika: Politische Pendelbewegungen, sozio-ökonomische Umbrüche und kulturelle Imaginarien in Geschichte und Gegenwart, Wien: Mandelbaum.

Burchardt, Hans-Jürgen (2021): Das pandemische Manifest. Neun Schritte in eine zukunftsfähige Gesellschaft. Oekom: München, [https://kassel-global.de/manife st/], letzter Aufruf 04.10.2021.

CEPAL (2012): Cambio estructural para la igualdad - Una visión integrada del desarrollo, Santiago: CEPAL.

CEPAL(2016): Panorama Social de América Latina y el Caribe 2015, Santiago: CEPAL.

CEPAL (2019a): Estudio Económico de América Latina y el Caribe, Santiago: CEPAL.

CEPAL (2019b): La autonomía de las mujeres en escenarios económicos cambiantes, Santiago: CEPAL.

CEPAL (2019c): Panorama Social de América Latina 2019, Santiago: CEPAL.

CEPAL (2020a): El desafío social en tiempos del COVID-19: COVID-19 Informe Especial, Santiago: CEPAL.

CEPAL (2020b): Anuario estadístico de América latina y el Caribe 2019, Santiago: CEPAL.

CEPAL (2021a): Revista CEPAL, 132, Santiago: CEPAL.

CEPAL/Oxfam (2019) Los incentivos fiscales a las empresas en América Latina y el Caribe, Santiago: CEPAL/Oxfam. 
CIAT (2018): Alianza para Generar Impacto: Informe anual CIAT, [https://ciat.cgiar.o rg/ar18/prologo/?lang=es], letzter Aufruf 07.09.2021

ECLAC (2018): Fiscal Panorama of Latina America and the Caribbean, Santiago: ECLAC.

Gutiérrez Cham, Gerardo, Herrera Lima, Susana; Kemner, Jochen (Hg.) (2021): Pandemia y crisis: El COVID19 en América Latina, Guadalajara: Editorial Universidad de Guadalajara.

Hecker, Paul (2020): Informalität und Ungleichheit im „linken“ Brasilien. Baden-Baden: Nomos.

ILO (2013): Panorama laboral 2013, Lima: International Labour Organisation.

ILO (2016): Políticas de formalización del trabajo doméstico remunerado en América Latina y el Caribe, Lima: International Labour Organisation.

ILO (2020a): Covid-19 crisis and the informal economy. Lima: International Labour Organisation.

ILO (2020ba): Organización Internacional del Trabajo, [https://www.ilo.org/americas/ sala-de-prensa/WCMS_735495/lang--es/index.htm], letzter Aufruf 07.09.2021.

Kessler, Gabriel; Brenza, Gabriela (2020): ¿Impactará la crisis del covid-19 en la agenda social de América Latina? in: Nueva Sociedad. [https://nuso.org/articulo /impactara-la-crisis-de-covid-19-en-la-agenda-social-de-america-latina/], letzter Aufruf 07.09.2021.

Lextartza, Larraitz; Chavez, María José; Carcedo, Ana (2016): Políticas de formalización del trabajo doméstico remunerado en América Latina y el Caribe, Lima: International Labour Organisation.

Martorano, Bruno (2018): Taxation and Inequality in Developing Countries: Lessons from the recent experience of Latina America, in: Journal International Development, 30, 256-273.

OECD, US \& CAF (2016): Perspectivas económicas de América Latina 2017: Juventud, competencias y emprendimiento, Paris: OECD.

OECD (2019): Estadística tributaria en América Latina y el Caribe, Paris: OECD.

OECD et al. (2021): Estadísticas tributarias en América Latina y el Caribe 2021, Paris: OECD.

Ruiz, Susana (2020): ¿Quien paga la cuenta? Gravar la riqueza para enfrentar la crisis de la COVID-19 en América Latina y el Caribe, Montreal: OXFAM International.

Weinmann, Nico (2020): Ungleichheitswirkung von Sozialreformen in Lateinamerika: Politische Regulierung bezahlter Haushaltsarbeit in Uruguay, Baden-Baden: Nomos.

WHO (2017). El mercurio y la salud, [https:/www.who.int/es/news-room/fact-sheets /detail/mercury-and-health\#: :text=El\%20mercurio\%20elemental\%20y\%20el,co n\%20consecuencias\%20a\%20veces\%20fatales], letzter Aufruf 07.09.2021. 
